\title{
Clinical Study Effectiveness of Maitland Techniques in Idiopathic Shoulder Adhesive Capsulitis
}

\author{
Abhay Kumar, ${ }^{1}$ Suraj Kumar, ${ }^{1}$ Anoop Aggarwal, ${ }^{2}$ Ratnesh Kumar, ${ }^{1}$ and Pooja Ghosh Das ${ }^{3}$ \\ ${ }^{1}$ Physiotherapy Department, CRC (Conposite Rehabilitation Center) Patna, Indian Red Cross Building, Near Gandhi Maidan, \\ Patna 800004, India \\ ${ }^{2}$ Physiotherapy Department, PDDUIPH (Pandit Deendayal Upadhyaya Institute for the Physically Handicapped), \\ 4 Vishnu Digamber Marg, New Delhi 110002, India \\ ${ }^{3}$ Physiotherapist, NIOH (National Institute for Orthopedically Handicapped), BT Road, Bon Hooghly, Kolkata 700090, India
}

Correspondence should be addressed to Suraj Kumar, surajdr2001@yahoo.com

Received 6 September 2012; Accepted 3 October 2012

Academic Editors: P. Czarnecki and K. Nas

Copyright (C 2012 Abhay Kumar et al. This is an open access article distributed under the Creative Commons Attribution License, which permits unrestricted use, distribution, and reproduction in any medium, provided the original work is properly cited.

\begin{abstract}
Objective. To study the effectiveness of Maitland techniques in the treatment of idiopathic shoulder adhesive capsulitis. Methods. total of 40 patients diagnosed with idiopathic shoulder adhesive capsulitis were recruited and randomly allocated into two groups. In Group A $(n=20)$ subjects were treated with Maitland mobilization technique and common supervised exercises, whereas subjects in Group B $(n=20)$ only received common supervised exercises. Variables. Shoulder pain and disability index (SPADI), VAS and shoulder ROM (external rotation and abduction) were variables of the study. These were recorded before and after the session of the training. Total duration of the study was four weeks. Result. Statistical analysis of the data revealed that within-group comparison both groups showed significant improvement for all the parameters, whereas between-group comparison revealed higher improvement in Group A compared to the Group B. Conclusion. The study confirmed that addition of the Maitland mobilization technique with the combination of exercises have proved their efficacy in relieving pain and improving R.O.M. and shoulder function and hence should form a part of the treatment plan.
\end{abstract}

\section{Introduction}

The term "capsulitis" or "frozen shoulder" referes to a common shoulder condition characterized by the global restriction in the shoulder range of motion in a capsular pattern. The capsular pattern in the shoulder is characterized by most limitation of passive lateral rotation and abduction [1]. The presence of capsular pattern is necessary to give a diagnosis of shoulder Capsulitis [2]. Although the ROM varies depending upon which stage the patient presents, yet he or she still has limitations of passive ROM in a capsular pattern. This condition was first described by Duplay [3] who called it "periarthrite scapulo-humerale." Codman [4] first introduced the term "frozen shoulder" and described it as a "class of cases which are difficult to define, difficult to treat and difficult to explain from the point of view of pathology" [5]. Neviaser [5] called it adhesive capsulitis, as he, under arthroscopy, observed that the capsule looked thickened and adhered to underlying bone and could be peeled off from the bone.

In an idiopathic capsulitis there is no apparent cause. The shoulder gradually becomes painful and stiff. Some authors have divided frozen shoulder in primary frozen shoulder, which corresponds to idiopathic. The secondary corresponds to traumatic capsulitis or if some other medical condition is present alongside [6]. The natural course of the condition is longer than generally stated and not always complete, that is, not all get full recovery.

The traditional principles of treatment of adhesive capsulitis are to relieve pain, maintain range of motion, and ultimately to restore function. The treatment of adhesive capsulitis by means of physiotherapy all along consists of different modalities (e.g., exercises, electrotherapy or massage) which may be applied side by side. Relief of pain may be achieved by massage, deep heat, ice, ultrasound, TENS (transcutaneous electrical nerve stimulation), and 
LASER (light amplification by stimulated emission of radiations) as described in our standard text books and other literature concerning the treatment of adhesive capsulitis. However, they probably offer little benefit [6-10]. Mostly these applications are adjunct to other treatment modalities like mobilization techniques or home exercise program [613].

Although adhesive capsulitis is generally considered to be a self-limiting condition that can be treated with physical therapy $[14,15]$, to regain the normal extensibility of the shoulder capsule, passive stretching of the shoulder capsule in all planes of motion by means of mobilization techniques has been recommended $[7,8,11,12]$.

The international Maitland Teachers Association (IMTA) defines the Maitland concept as a process of examination, assessment, and treatment of neuromusculoskeletal disorder by manipulative physiotherapy [12].

Grades I and II of Maitland mobilization techniques are primarily used for treating joints limited by pain. The oscillations may have an inhibitory effect on the perception of painful stimuli by repetitively stimulating mechanoreceptors that block nociceptive pathways at the spinal cord or brain stem levels. These nonstretch motions help move synovial fluid to improve nutrition to the cartilage whereas Grades III and IV are primarily used as stretching manoeuvres. Appropriate selection of mobilization technique for treatment can only take place after a thorough assessment and examination.

As mentioned above, the capsulitis is challenging for therapeutic as well as rehabilitation purposes. In this present work, the purpose is to evaluate the efficacy of the Maitland mobilization in the rehabilitation of the adhesive capsulitis. It is hypothesized that the importance of Maitland techniques is more effective than the conventional exercise program in case of adhesive capsulitis.

\section{Materials and Methods}

Forty subjects (both male and female) between age group of 40 and 60 were selected from National Institute for the Orthopaedically Handicapped (NIOH) Outpatient Department after they were diagnosed of suffering from the idiopathic shoulder adhesive capsulitis. All subjects were diagnosed by orthopedic doctor and they were checked for the global restriction at shoulder joint, by expert physiotherapist. After the initial assessment, written informed consent forms were obtained from the participants who met the inclusion criteria. The inclusion criteria of the study were age between 40 to 60 years; shoulder ROM restriction; pain more than 2 months. All the patients were having global restriction of shoulder joint range of motion, that is, movements of shoulder were restricted in shoulder in all direction. Subjects were excluded if they had history of fracture around shoulder; any inflammatory disorder around shoulder; diabetic or any neurological involvement; patient taking any steroids and/or analgesics; having any cardiac conditions. Figure 1 shows the methodology adopted in the study. Since the symptoms of patients were more than two months old, therefore they were not given any medical intervention in form of steroidal injection, or NSAID (nonsteroidal anti inflammatory drugs).

The selected candidates were randomly allocated to two different groups: experimental (Group A) and control (Group B) each having 20 participants. The randomization was done using a chit pick box method. One box contained 20 chits labeled "group-A" and 20 chits labeled "group-B". Whenever patient was selected for study, a chit was picked from the box, and whatever chit was picked, the patient was assigned to that group.

Variables of this study included VAS (Visual analogue scale) for pain, SPADI for functional outcome measures, and two ROM selected for the study, that is, Shoulder external rotation and shoulder abduction. Abduction range and external rotation range were measured by goniometer. Each subject was asked to mark on the $10 \mathrm{~cm}$ long visual analogue scale (VAS) for pain intensity and is also requested to complete the shoulder pain and disability index (SPADI) Questionnaire. After the assessment and the data collection, participants were given the therapeutic intervention according to their groups. The whole procedure was approved from the Institutional Ethical Committee (IEC) of NIOH.

Two groups pretreatment-posttreatment test design was done, and study period of this study was 4 weeks.

\subsection{Interventions}

2.1.1. Maitland Mobilization. Experimental Group A $(n=$ 20) was treated with Maitland's mobilization techniques (Figure 2) and supervised exercises. The glides given included glenohumeral caudal glide, glenohumeral caudal glide progression; glenohumeral postero-anterior glide. Passive oscillatory movements were performed at the rate of 2-3 glides per second for 30 seconds for each glide and every glide was given for 5 sets. The technique was applied thrice a week for four weeks (12 sessions).

2.1.2. Common Supervised Exercises Program. This intervention was given to the experimental as well as the control group. Supervised exercise program was explained and patients were required to repeat all these exercises at center under supervision of the therapist (Figure 3 ).

Intervention consisted of the Codman exercise [4], shoulder wheel exercises [6], self-stretching exercises [6], wall-ladder exercises $[6,16]$, and self-stretching exercises (for improving abduction, flexion, external rotation, internal rotation, and horizontal adduction).

For improving the abduction, patient was sitting with the side next to a table, the forearm resting with palm up and patient was asked to slide his or her arm across the table, remaining in this position for 10 seconds, relax in starting position, and repeat it for 10 times.

Similarly for improving the flexion the client was asked to slide the forearm forward along the table, remain in this position for 10 seconds, relax in starting position, and repeat it for 10 times. For improving the lateral rotation, the client stood standing and facing a doorframe with the palm of the hand against the edge of the frame and elbow flexed 90 . While 


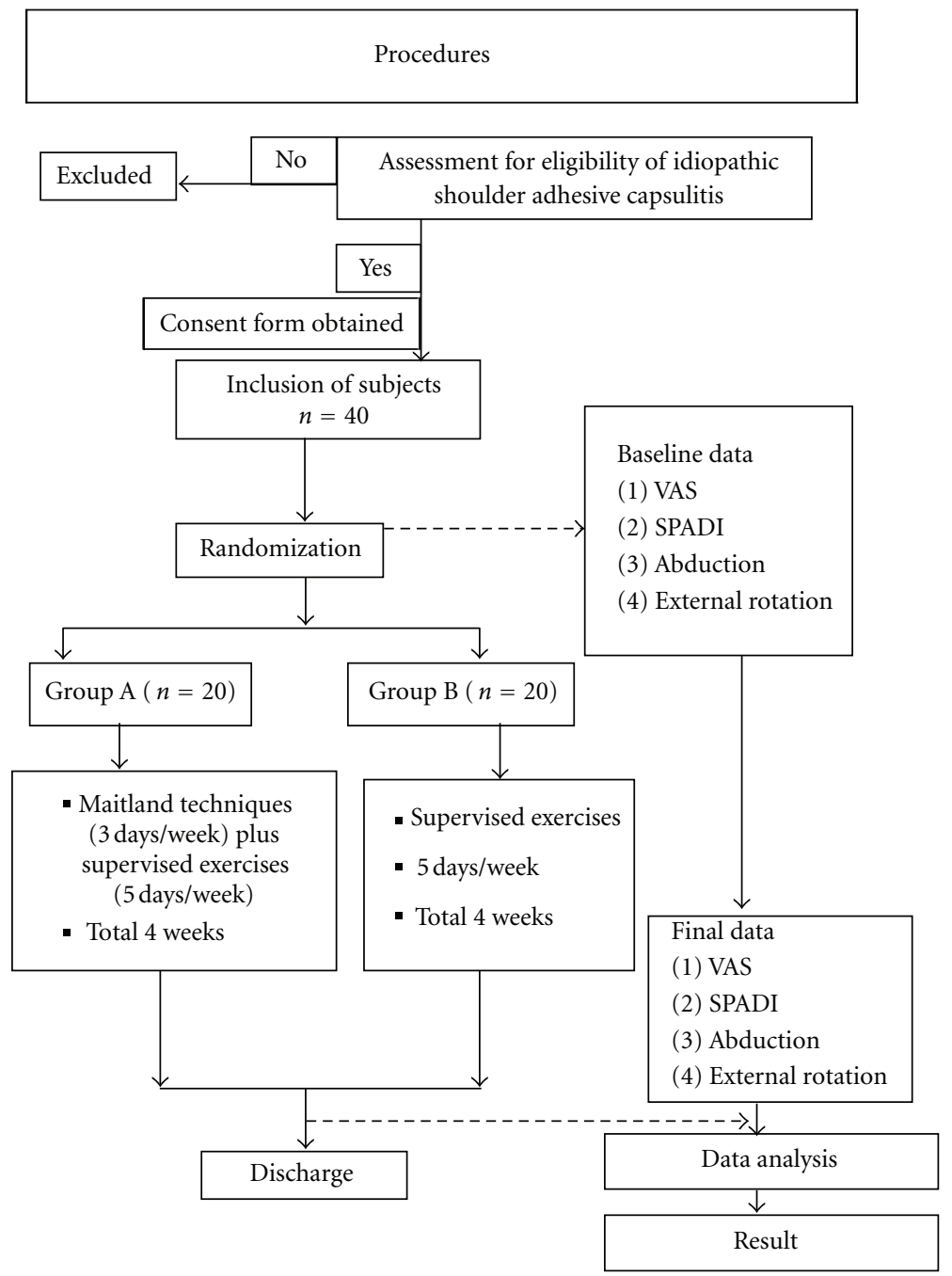

Figure 1: Flow chart of methodology.

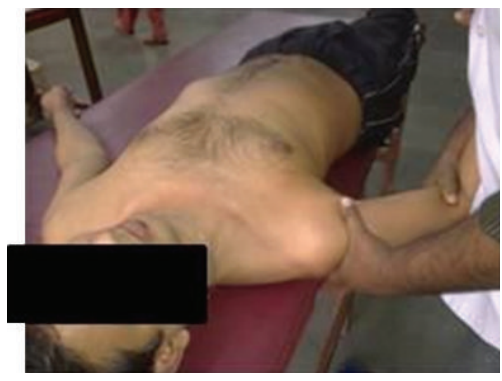

(a) Caudal glide initial position

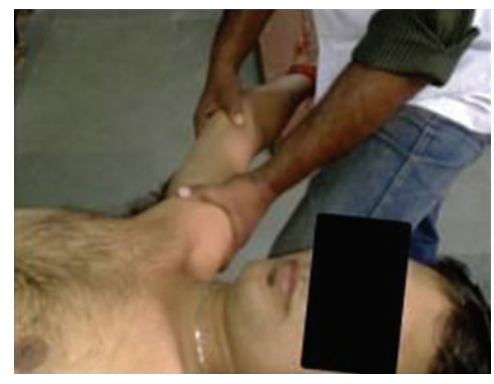

(b) Caudal glide final position

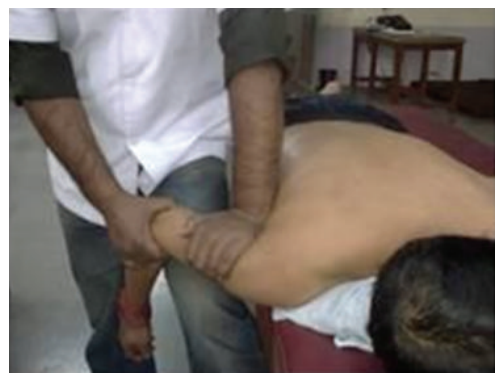

(c) Glenohumeral joint PA glide

Figure 2: Maitland mobilization for giving Caudal glide (a) Initial position and at (b) final position; (c) PA posterio-anterior glide of gleno-humeral joint.

keeping the arm against the side or in slight abduction, the subjects were asked to turn away from the fixed hand, remain in this position for 10 seconds, relax in starting position, and repeat it for 10 times.

For improving the medial rotation, the client remained in standing or in high sitting with holding the towel from back by using his or her both hand. Subjects hold the towel from back of the neck through affected side hand and tried to pull the towel from sound arm hand through lower back, hold it for 10 seconds, and relax, and Repeat it for 10 times.

To improve the shoulder extension, the client stood with the back to the table. Both hands were grasping the edge with 


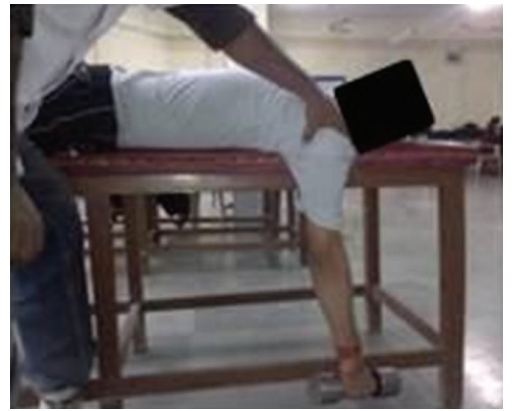

(a)

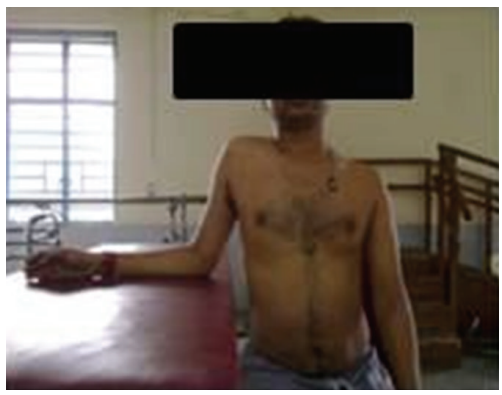

(d)

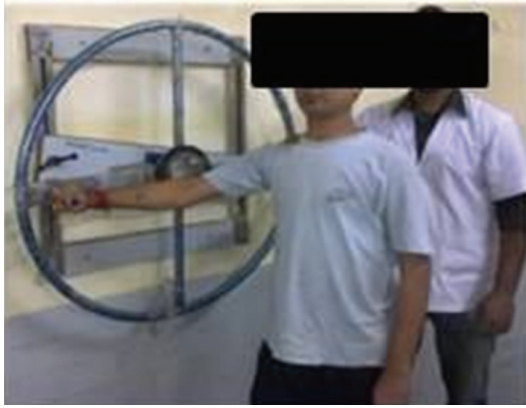

(b)

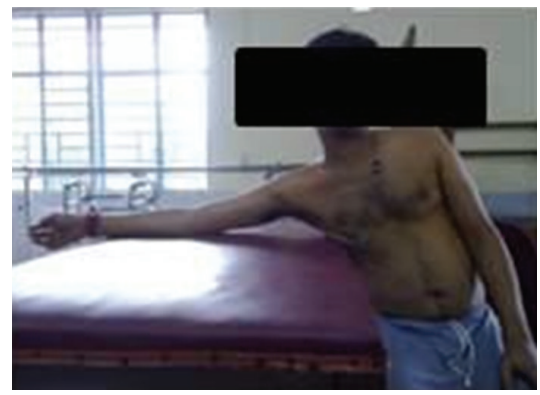

(e)

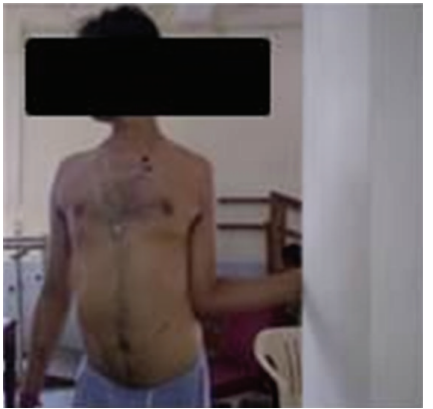

(c)

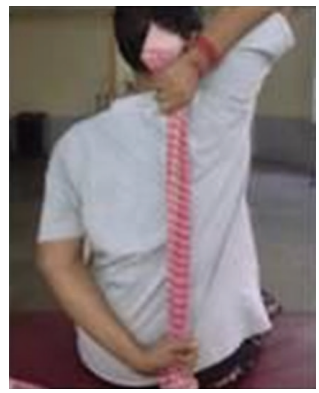

(f)

FIgURE 3: Supervised exercise program. (a) Codman's exercises; (b) shoulder wheel; ((c) and (d)) to increase shoulder external rotation; (e) to increase shoulder abduction; (f) to increase shoulder medial rotation.

the fingers facing forward. The subjects were asked to begin to squat while letting the elbows flex, hold it for 10 seconds, and relax, repeat it for 10 times. To increase the horizontal adduction clients performed cross stretch where they were asked to adduct the tight shoulder horizontally by placing the arm across the chest. Apply sustained overpressure to the adducted arm by pulling the arm toward the chest.

2.2. Data Analysis. SPSS version 15 software was used for analysis of the gathered data. "Paired sample $t$-test" was done to analyze within-group variables for VAS and SPADI. "Independent sample $t$-test" was done to analyze betweengroups variables for VAS and SPADI. External rotation and abduction range were analyzed by Wilcoxon Signed Ranks test for within-group and Mann-Whitney $U$ test for betweengroup variables. The $P$ value was set at $<0.05$.

\section{Results}

3.1. Demographic Data. Total of 40 patients (female $=14$ and male $=26)$ participated in the study. Their variables had insignificant difference between the two groups at preintervention levels (Table 1).

3.2. Pain Intensity. In both groups, that is, Group A and Group B, there is a statistically significant difference between VAS and VAS4 $(P<0.05)$ between pretest and posttest scores. The mean improvement in Group A was $5.99 \pm 0.01$ and in Group B was $5.19 \pm 0.04$ (Table 2). Both groups have shown statistically significant improvement in pain scores.
TABle 1: Patient demographics.

\begin{tabular}{lccc}
\hline Variables & Group-A & Group-B & Total \\
\hline$n=$ no. of patients & 20 & 20 & 40 \\
Age (mean) & 47.9 & 47.1 & 47.5 \\
\hline
\end{tabular}

During between-group comparison, it was observed that the baseline characteristics of the data for both groups, that is, VAS0 is statistically insignificant $(P=0.885)$. The posttreatment reading at the end of 4 th week (VAS4) was found to be statistically significant between the two groups $(P=0.005)$ (Table 2 and Figure 4$)$, and Group A has shown more improvement than Group B.

3.3. Shoulder Pain and Disability Index (SPADI). In both groups, that is, Group A and Group B there was a statistically significant difference between SPADI0 and SPADI4 $(P<$ $0.05)$. The mean improvement in Group $A$ is $40.83 \pm 3.89$ and in Group B is $36.34 \pm 1.97$ (Table 3). Both groups have shown statistically significant improvement in SPADI scores.

During between-group comparison, it was observed that the baseline characteristics of the data for both groups, that is, SPADI0, was statistically insignificant $(P=0.539)$. The posttreatment reading at the end of 4th week (SPADI4) was found to be statistically significant between the two groups $(P=0.005)$ (Table 3$)$, and Group A has shown more improvement than Group B (Figure 4).

3.4. External Range of Motion. In both groups, that is, Group A and Group B, there was a statistically significant 


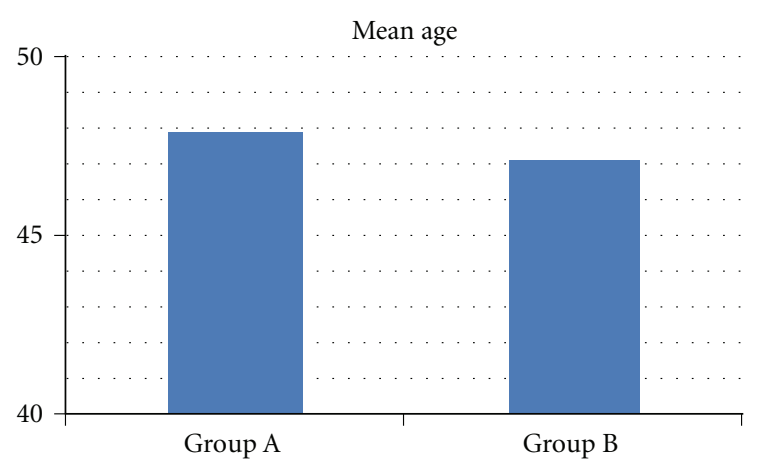

Mean age

(a)

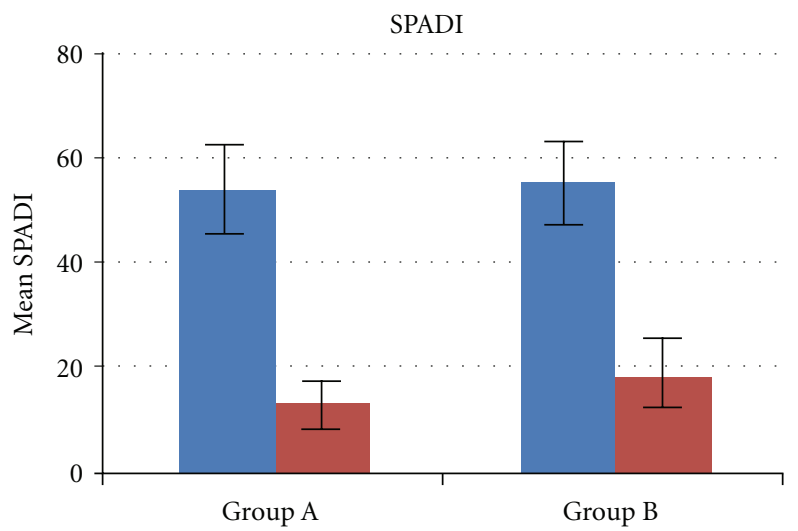

SPADI0

SPADI4

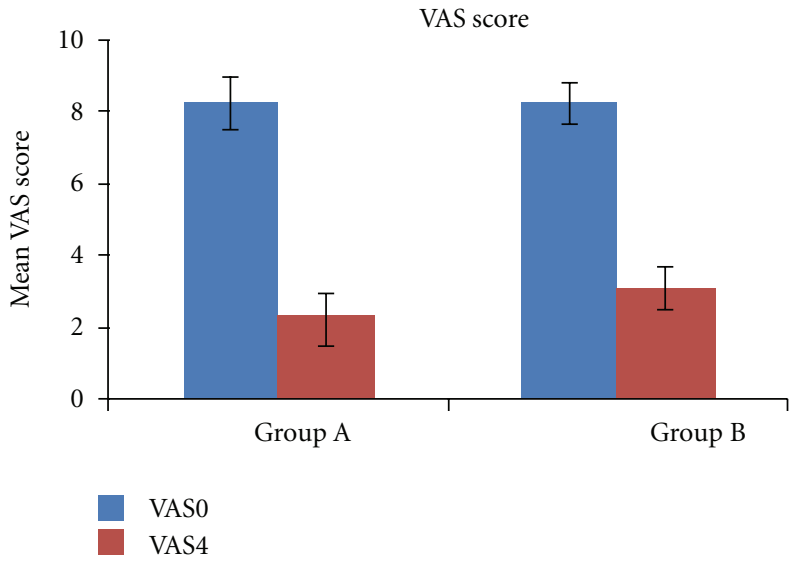

(b)

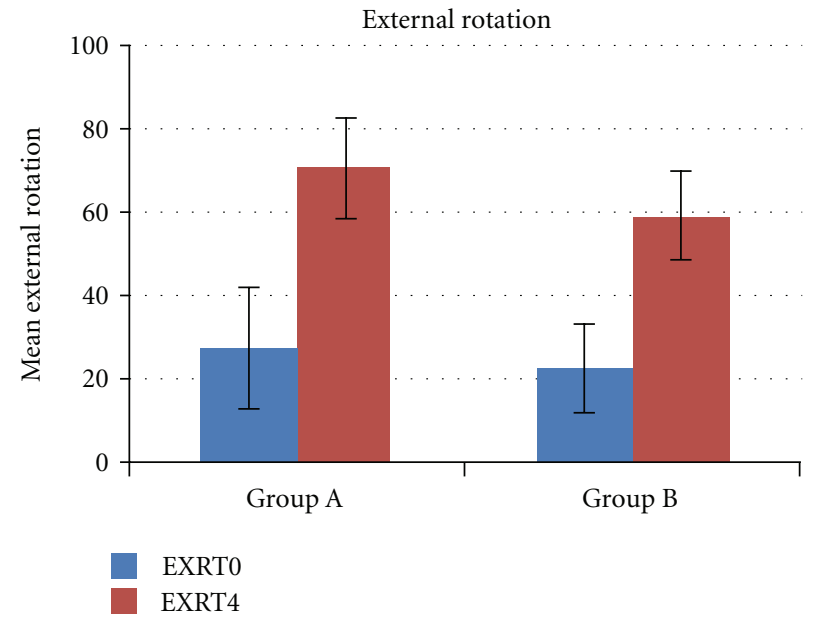

(d)

(c)

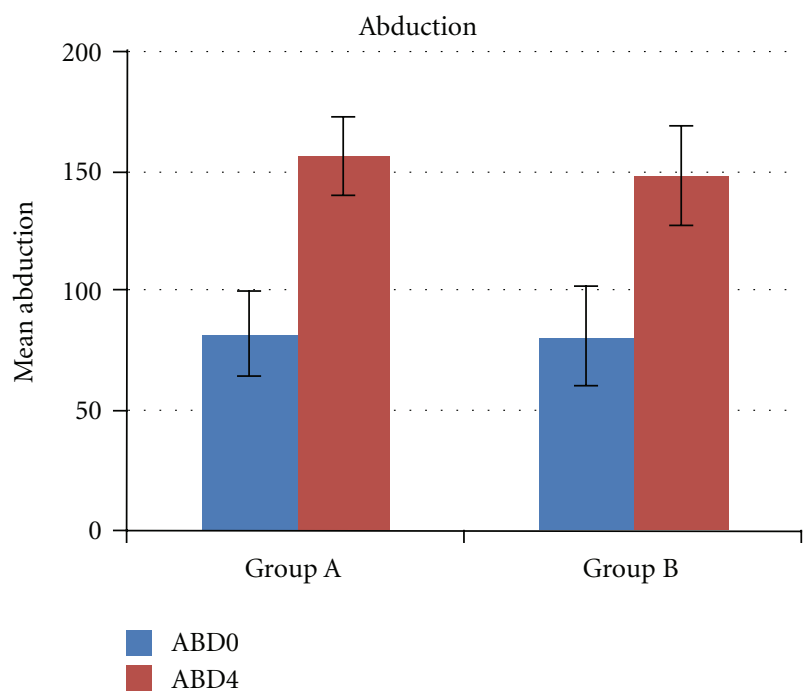

(e)

FIGURE 4: Graphical representation of between-group and within group comparison of variables. VAS: visual analogue scale; SPADI: shoulder pain and disability index; 0: preteting score; 4: post-testing score. 
TABLE 2: Analysis of VAS scores.

\begin{tabular}{lccc}
\hline & VAS scores & \multicolumn{2}{c}{ Within-group analysis } \\
& VAS0 & VAS4 & $t$ \\
Group A & $8.22(0.74)$ & $2.23(0.73)$ & 29.267 \\
Group B & $8.25(0.54)$ & $3.06(0.58)$ & 38.35 \\
$T$ & -0.145 & -3.975 & 0.005 \\
$P$ & 0.885 & 0.005 & \\
& Between-group analysis & & \\
\hline
\end{tabular}

TABLE 3: Analysis of SPADI scores.

\begin{tabular}{|c|c|c|c|c|}
\hline \multicolumn{3}{|c|}{ SPADI scores } & \multicolumn{2}{|c|}{ Within-group analysis } \\
\hline & SPADI0 & SPADI4 & $T$ & $P$ \\
\hline Group A & $53.68(8.61)$ & $12.85(4.72)$ & 21.4 & 0.005 \\
\hline Group B & $55.3(7.93)$ & $18.96(5.96)$ & 15.32 & 0.005 \\
\hline$T$ & -0.620 & -3.587 & & \\
\hline \multirow[t]{2}{*}{$P$} & 0.539 & 0.001 & & \\
\hline & Between-group a & & & \\
\hline
\end{tabular}

difference between EXRT0 and EXRT4 $(P<0.05)$. The mean improvement observed in Group $\mathrm{A}$ is $42 \pm 1.81$ and in Group B is $36.25 \pm 0.13$ (Table 4 ). Both groups improved significantly in external range of motion.

For both groups, the baseline characteristics of the data, that is, EXRT0, is statistically insignificant $(P=0.336)$. The posttreatment reading at the end of 4 th week (EXRT4) was found to be statistically significant between two groups $(P=$ 0.001) (Table 4). Group A has shown better improvement in comparison to Group B (Figure 4).

3.5. Abduction Range of Motion. In both groups, that is, Group A and Group B, there was a statistically significant difference between ABD0 and ABD4 $(P<0.05)$. The mean improvement observed in Group A was 74.2 \pm 1.03 and in Group B is $61 \pm 2.02$ (Table 5). Both groups improved significantly in abduction range of motion.

For both groups, the baseline characteristics of the data, that is, ABD0, is statistically insignificant $(P=0.807)$. The posttreatment reading at the end of 4 th week (ABD4) was found to be statistically significant between two groups $(P=$ 0.001) (Table 5). Group A has shown better improvement in comparison to Group B (Figure 4).

\section{Discussion}

The present study was designed to know the efficacy of Maitland mobilization techniques adjunct with exercises in the treatment of idiopathic shoulder adhesive capsulitis by comparing with exercises alone.

While analyzing the outcome measures of this study, it was observed that both the groups have shown significant improvement over time. Statistical analysis of the data in pre- and postintervention VAS values illustrated difference (shown by decreasing trends in Table 2 and Figure 4(a) for both groups). Though both groups have significantly reduced pain scores, the difference was found in favor of Group A in between-group comparison (Table 2 and Figure 4). Both the groups shown reduction in pain scores, and this is in agreement with previous study suggesting that mobilization reduces pain [15] due to neurophysiologic effects on the stimulation of peripheral mechanoreceptors and the inhibition of nociceptors [17, 18]. The activation of apical spinal neurons as a result of peripheral mechanoreceptor by the joint mobilization produces presynaptic inhibition of nociceptive afferent activity [19].

Mechanical force during mobilization may include breaking up of adhesions, realigning collagen, or increasing fibre glide when specific movements stress the specific parts of the capsule [20]. Furthermore mobilization techniques are supposed to increase or maintain joint mobility by inducing biological changes in synovial fluid, enhanced exchange.

Maitland's mobilization mainly consists of rhythmic oscillatory movements which stimulate the type- 2 dynamic mechanoreceptors and by this way can inhibit the type- 4 nociceptive receptors [11].

Maitland's rhythmic oscillations also has an effect on circulatory perfusion. The ongoing circulatory stasis may lead to ischemia and the potential for intraneural oedema, inflammation, and fibrosis. Mobilization has an effect on fluid flow as blood flow in the vessels supplying the nerve fibres and synovial fluid flow surrounding the avascular articular cartilage. This, by a pressure gradient, is generated which helps in facilitating exchange of fluid, that is, increased venous drainage and dispersing the chemical irritants. This causes a reversal of the ischemia, edema, and inflammation cycle and reduces joint effusion and relieves pain by reducing the pressure over the nerve endings. 
TABLE 4: Analysis of external rotation scores.

\begin{tabular}{lcccc}
\hline & External rotation ROM & & \multicolumn{2}{c}{ Within-group analysis (Wilcoxon test) } \\
& ER0 & ER4 & $t$ & 0.005 \\
Group A & $27.25(14.63)$ & $69.25(12.82)$ & -3.92 & 0.005 \\
Group B & $22.5(10.54)$ & $58.75(10.67)$ & -3.92 & \\
$U$ & -0.962 & -3.295 & \\
$P$ & 0.336 & 0.001 & & \\
& \multicolumn{2}{c}{ Between-group analysis (Mann-Whitney test) } \\
\hline
\end{tabular}

TABLE 5: Analysis of abduction scores.

\begin{tabular}{lccc}
\hline & Abduction ROM & & \multicolumn{2}{c}{ Within-group analysis } \\
& ABD0 & ABD4 & $t$ \\
Group A & $82.05(17.57)$ & $156.25(16.54)$ & -3.923 \\
Group B & $81.05(21.25)$ & $142.50(19.23)$ & -3.922 \\
$T$ & -0.245 & -3.065 & 0.005 \\
$P$ & 0.807 & 0.002 & \\
& Between-group analysis & & \\
\hline
\end{tabular}

The neurophysiologic effect is based on the stimulation of peripheral mechanoreceptors and inhibition of nociceptors [12].

In Group B, noticeable improvement may be due to beneficial effect of supervised exercise protocol. Many studies have claimed that exercise programme is the most effective treatment for shoulder adhesive capsulitis [21].

Exercises within the pain free range of motion stimulates mechanoreceptors and decreases pain. Exercises within pain free range also move the synovial fluid, thus decrease inflammation and decreased pain $[22,23]$.

Both groups received Codman's exercises. Weight was not used if pain was severe. This techniques uses the effects of gravity to distract the humerus from the glenoid fossa. They help to relieve pain through gentle traction and oscillation and provide movement of the synovial fluid. It also relives pain through the neurophysiological and mechanical effect [6].

Both groups improved significantly in range of motion of external rotation.

4.1. Abduction Ranges. Both groups improved significantly in abduction range of motion. And Group A (Maitland mobilization) shows a statistically significant improvement in ROM than Group B.

Considering the pathology of adhesive capsulitis wherein there is a contracture of joint capsule along with other periarticular structures, the effects of Maitland's mobilizations allow for stretching of the shortened and contracted soft tissues, besides providing the necessary translational movements required to gain the normal physiological movements of the shoulder thus inducing beneficial effects.

The predominant adhesive capsule and associated soft tissue tightness of frozen shoulder have been commonly addressed in clinical treatment approaches by mobilization techniques [24]. Mobilization techniques improve the normal extensibility of the shoulder capsule and stretch the tightened soft tissues to induce beneficial effects. It may be attributed to the fact that the intent of end-range mobilizations is not only to restore joint play but also to stretch contracted periarticular structures [24].

Whereas Group A received mobilization, gliding for mobilization selected to increase external range of motion was postero-anterior glide and to increase abduction, caudal glide was selected. It may be the reason that postero-anterior glide and caudal glide of the glenohumeral joint increased the capsular extensibility and lengthen the soft tissues which were inhibiting joint play movement at the joint. So this increased capsular extensibility may have permitted increased range of motion at the glenohumeral joint. These techniques also thought to increase the proprioceptive and kinesthetic sensation in the joint thus individuals can do the activities in newly gained range of motion. So person can maintain the improved range of motion by using the joint appropriately. This result supports the findings of previous studies showing improvement after mobilization in adhesive capsulitis $[24,25]$.

Both groups received several stretching exercises, so this can be reason for improvement in both groups. It has been proved that stretching exercises increases the extensibility of the soft tissue on the basis of creep response thus altering the viscoelastic properties and range of motion can be gained. Individuals need to do the activities in newly gained range of motion to maintain motion at the joint. This result supports the findings of previous studies showing improvement after exercises in adhesive capsulitis [26].

Group A received mobilization additionally so this may be the reason of greater improvement compare to Group B.

4.2. SPADI Score. Both groups have shown statistically significant improvement in shoulder pain and disability index score (SPADI) proving the improvement in shoulder 
function in both groups after having undergone mobilization.

This result correlates with previous studies $[24,26]$ which studied the effects of Maitland's ERM and exercises on subjects of adhesive capsulitis and found that besides pain and ROM function also improved.

Rationale behind improvement in functional capacity might be due to ease in pain and increased range of motion, consequently lessened suffering in daily activities, pain with specific tasks, and difficulty in moving arm and lifting actions. When patient's pain decreased, it revealed a reduction in SPADI scores. Both groups had reduction in their pain and improved their range of motion so this could be the reason that both groups revealed a reduction in their SPADI scores. Whereas Group A improved better in terms of pain and range of motion, the group $B$ had better reduction in the SPADI scores.

Stasinopoulos [27] accounted that the treatment regimen of supervised exercise programme should be at least three times per week for four weeks and home exercise for at least three months [8].

Results of this study after analysis were directed towards the conclusion that Maitland mobilization technique with supervised exercise protocol more effective for treating idiopathic shoulder adhesive capsulitis, as the experimental group (Group A) has shown significant improvement than the control group (Group B) in all outcome parameters. The results were significant at $P<0.05$ with $95 \%$ confidence interval in between the groups for pain intensity, SPADI score, and the range of motion of abduction and external rotation of shoulder. Thus, these results allowed rejection of the null hypothesis and thereby supported to accept the experimental hypothesis. These results strongly support the earlier findings of study [16] that investigated the shoulder motion pain and function by using mobilization and exercises on single case design and concluded that all four movements improved, although more gain in motion was observed when mobilizations were added.

\section{Conclusion}

This study found that mobilization of the shoulder must be added to the supervised exercise program to achieve goals of reducing pain, improving $\mathrm{ROM}$, and function, in adhesive capsulitis.

\section{References}

[1] Cyriax, Text Book of Orthopaedic Medicine, vol. 1, Oxford, UK, 8th edition, 1982.

[2] J. D. Magee, Orthopedic Physical Assessment, Saunders, 4th edition, 2002.

[3] E. S. Duplay, "De la periarthrote scapulohumerale," General Medicine, vol. 20, pp. 513-542, 1872.

[4] Codman, The Shoulder, Yhomas Todd Company, Boston, Mass, USA, 1934.

[5] J. S. Neviaser, "Adhesive capsulitis of shoulder," The Journal of Bone \& Joint Surgery, vol. 27, pp. 211-222, 1945.

[6] C. Kisner, Lynn Allen Colby, Therapeutic Exercises, 4th edition, 2002.
[7] J. Mennel, Joint Pain. Diagnosis. Treatment Using Manipulative Techniques, Litlle, Boston, Mass, USA, 1964.

[8] F. M. Kaltenborn, Manual Therapy for the Extremity Joints, Olaf Norlis Bokhandel, Oslo, Norway, 2nd edition, 1976.

[9] S. Brotzman and E. Kevin Wilk, Clinical Orthopedic Rehabilitation, Mosby, Philadelphia, Pa, USA, 2nd edition, 2003.

[10] W. Munrray, "The chronic frozen shoulder," Physical Therapy Review, vol. 40, p. 866, 1960.

[11] G. D. Maitland, "Treatment of the glenohumeral joint by passive movement," Physiotherapy, vol. 69, no. 1, pp. 3-7, 1983.

[12] G. D. Maitland, Peripheral Manipulation, Butterworth- Heinemann, 2nd edition, 1991.

[13] W. Ben Kibler, "Shoulder rehabilitation: principles and practice," Medicine and Science in Sports and Exercise, vol. 30, no. 4, pp. S40-S50, 1998.

[14] J. Ozaki, Y. Nakagawa, G. Sakurai, and S. Tamai, "Recalcitrant chronic adhesive capsulitis of the shoulder. Role of contracture of the coracohumeral ligament and rotator interval in pathogenesis and treatment," Journal of Bone and Joint Surgery A, vol. 71, no. 10, pp. 1511-1515, 1989.

[15] F. A. Simmonds, "Shoulder pain with particular reference to the frozen shoulder," The Journal of Bone and Joint Surgery, vol. 31, no. 3, pp. 426-432, 1949.

[16] N. Maricar, C. Shacklady, and L. McLoughlin, "Effect of Maitland mobilization and exercises for the treatment of shoulder adhesive capsulitis: a single-case design," Physiotherapy Theory and Practice, vol. 25, no. 3, pp. 203-217, 2009.

[17] B. W. Wyke, "Articular neurology—a review," Physiotherapy, vol. 58, no. 3, pp. 94-99, 1972.

[18] W. H. Akeson, D. Amiel, and S. L. Y. Woo, "Immobility effects on synovial joints the pathomechanics of joint contracture," Biorheology, vol. 17, no. 1-2, pp. 95-110, 1980.

[19] B. W. Wyke, "Articular neurology-a review," Physiotherapy, vol. 58, no. 3, pp. 94-99, 1972.

[20] R. Donatelli and J. M. Wooden, Orthopaedic Physical Therapy, Churchill Levingstone, New York, NY, USA, 2nd edition, 1994.

[21] T. J. Neviaser, "Adhesive capsulitis," Orthopedic Clinics of North America, vol. 18, no. 3, pp. 439-443, 1987.

[22] A. K. Biswas, B. N. Sur, and C. R. Gupta, "Treatment of periarthritis shoulder," Journal of the Indian Medical Association, vol. 72, no. 12, pp. 276-277, 1979.

[23] H. C. Liang and I. N. Lien, "Comparative study in the management of frozen shoulder," Journal of the Formosan Medical Association, vol. 72, no. 4, pp. 243-247, 1973.

[24] H. M. Vermeulen, W. R. Obermann, B. J. Burger, G. J. Kok, P. M. Rozing, and C. H. M. Van den Ende, "End-range mobilization techniques in adhesive capsulitis of the shoulder joint: a multiple-subject case report," Physical Therapy, vol. 80, no. 12, pp. 1204-1213, 2000.

[25] H. M. Vermeulen, P. M. Rozing, W. R. Obermann, S. le Cessie, and T. P. Vliet Vlieland, "Comparison of high-grade and low-grade mobilization techniques in the management of adhesive capsulitis of the shoulder: randomized controlled trial," Physical Therapy, vol. 86, no. 3, pp. 355-368, 2006.

[26] J. F. Chen, K. A. Ginn, and R. D. Herbert, "Passive mobilisation of shoulder region joints plus advice and exercise does not reduce pain and disability more than advice and exercise alone: a randomised trial," Australian Journal of Physiotherapy, vol. 55, no. 1, pp. 17-23, 2009.

[27] D. Stasinopoulos and M. I. Johnson, "Cyriax physiotherapy for tennis elbow/lateral epicondylitis," British Journal of Sports Medicine, vol. 38, no. 6, pp. 675-677, 2004. 


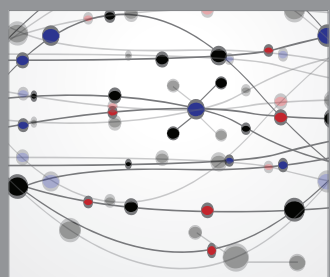

The Scientific World Journal
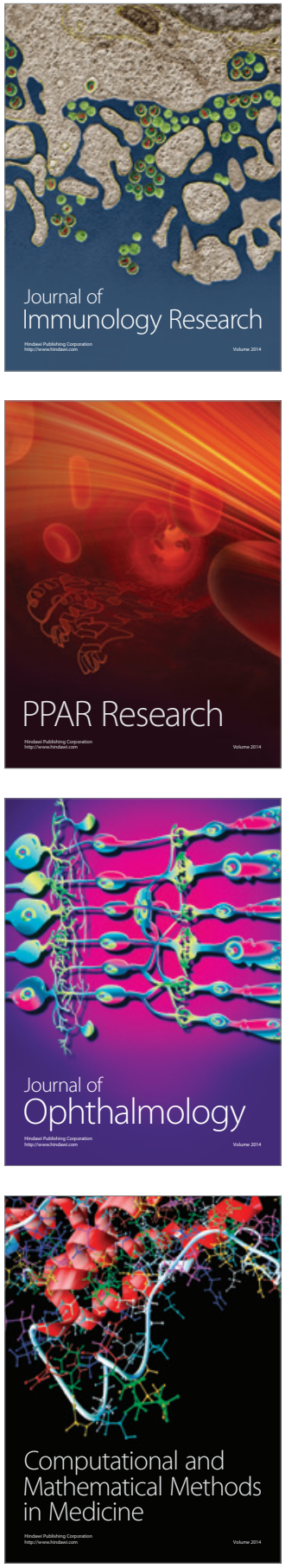

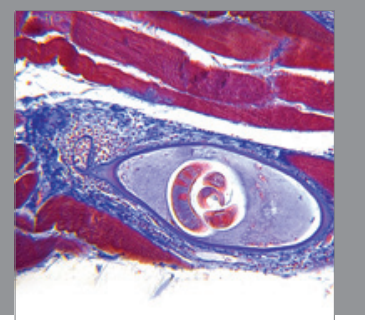

Gastroenterology

Research and Practice
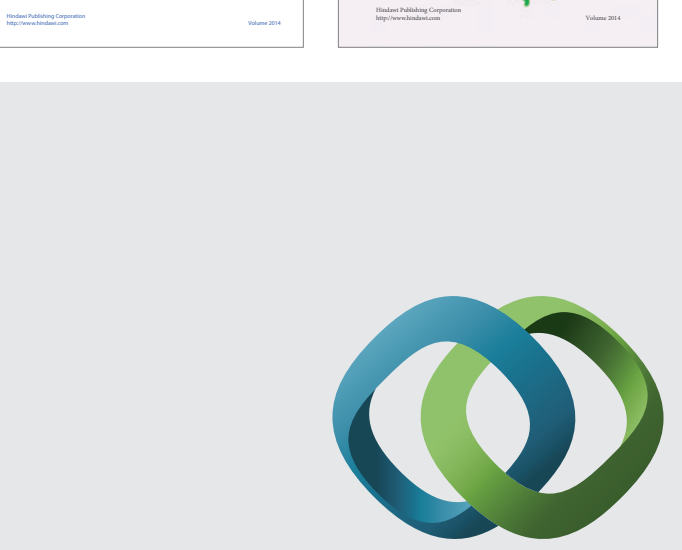

\section{Hindawi}

Submit your manuscripts at

http://www.hindawi.com
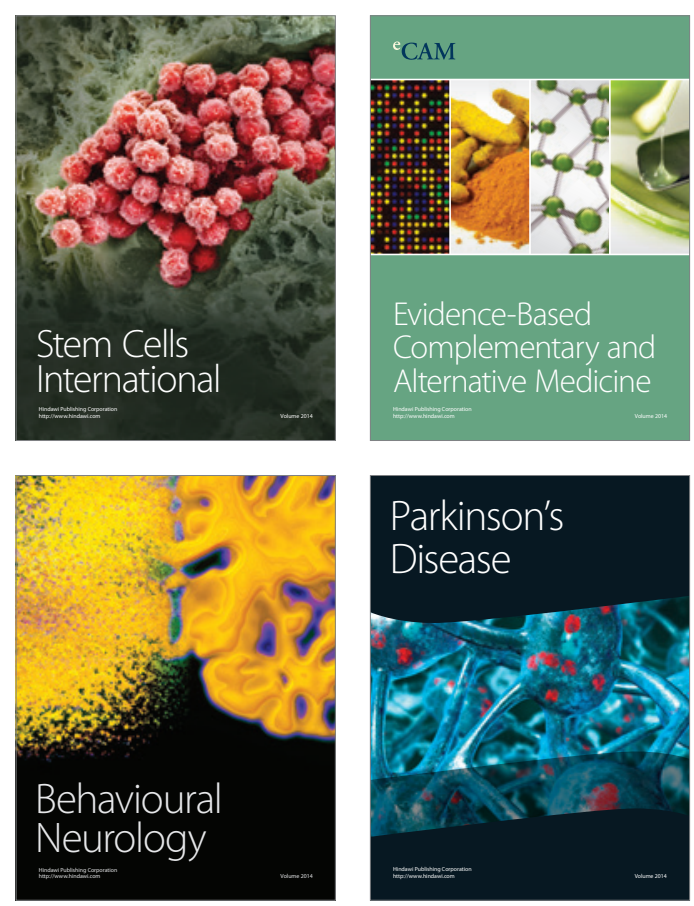

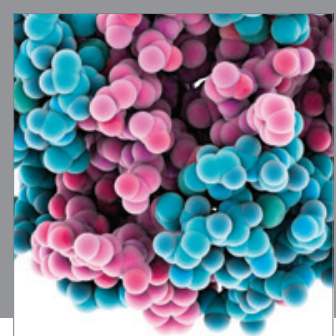

Journal of
Diabetes Research

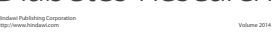

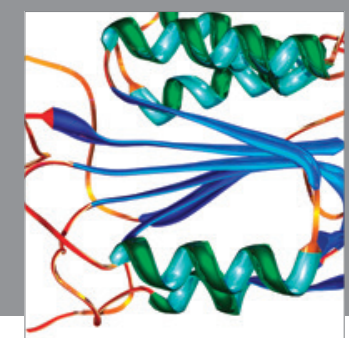

Disease Markers
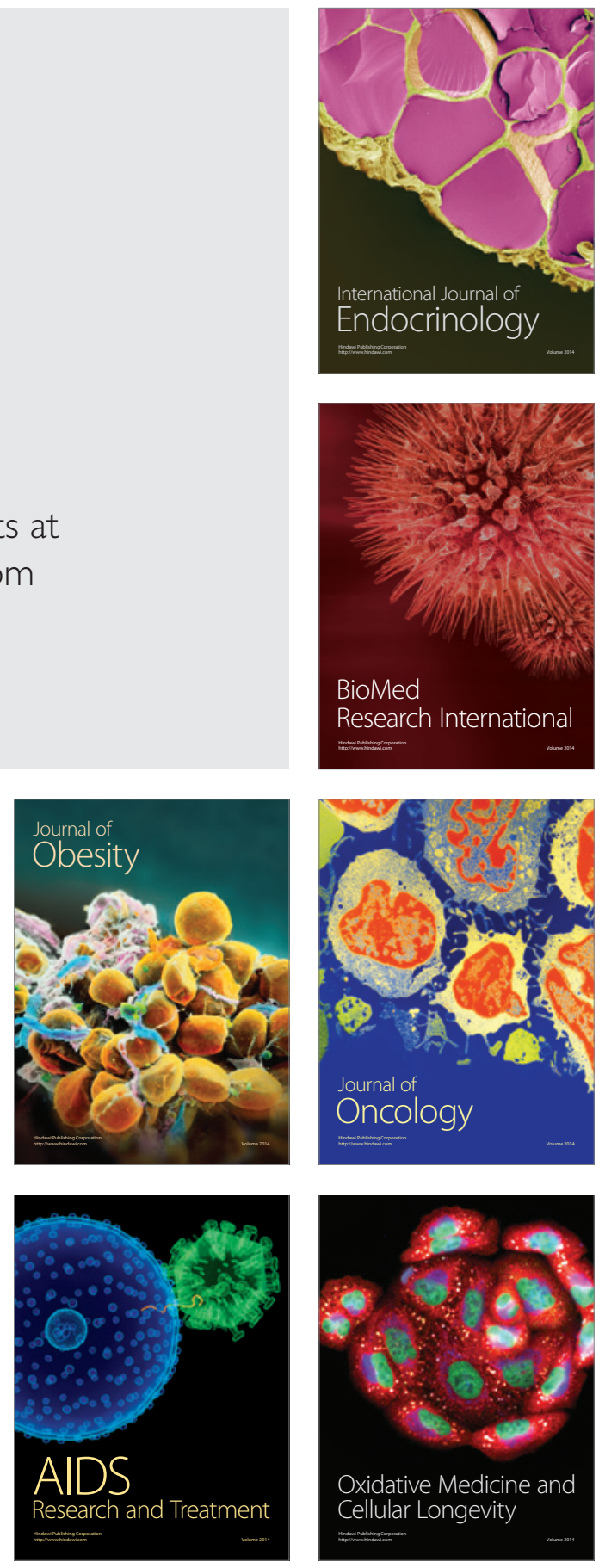